\title{
Nachhaltige Medizin oder nachhaltige Gesundheit?
}

Johannes G. Schmidt

Die ganze Welt ist krank, weil wir nur über Krankheiten reden und darob die Gesundheit vergessen haben. (Stiftung Paracelsus heute)

In ihrem Positionspapier «Nachhaltige Medizin» [1] stellt die Schweizerische Akademie der Medizinischen Wissenschaften die entscheidende Frage: $\mathrm{Ob}$ anstelle einer nachhaltigen Medizin nicht eine nachhaltige Gesundheit zu thematisieren sei? Nein, die Medizin sei auf das «Medizinische» begrenzt und Gesundheit sei eine Frage der «Politik» und der «Gesellschaft». Das im Ganzen sehr vielversprechende Papier zählt die Faktoren auf, die einer nicht nachhaltigen «Maximalmedizin» Vorschub leisten. Ohne Gesundheit als Thema geht jedoch der Grund hinter all diesen Faktoren unter: Ein medizinisches Denken, das sich verirrt hat in der Täuschung, Gesundheit entstehe (nur) durch die Bekämpfung und Beseitigung von Krankheiten.

\section{«Gesunde und von selber Gesundende werden in wach- sendem Ausmass durch die Medizin krank gemacht.»}

Korrespondenz:

Dr. med. Johannes G. Schmidt Praxiszentrum Meinradsberg Stiftung Paracelsus heute Ilgenweidstrasse 3 CH-8840 Einsiedeln

schmidt[at]paracelsus-heute.ch

\section{Wo bleiben Gesundheit und Salutogenese?}

Die Diskussion einer nachhaltigen Gesundheit braucht keine Definition der (absoluten) Gesundheit, denn in Praxis und Alltag ist klar, was gemeint ist. Ist die Gesundheit beeinträchtigt? Hat sie sich verändert? Lässt sie sich verbessern, nachhaltig? Die (relative) Gesundheit ist immer Gegenstand der Medizin gewesen, mit der evidenzbasierten Medizin ist sie sogar wieder ausdrücklich ins wissenschaftliche Blickfeld gerückt - mit der systematischen Frage nach Erfolgskriterien und Endpunkten, die eine echte und klinisch relevante Aussage über den gesundheitlichen Nutzen erlauben. Allerdings befasst sich die Medizin weiterhin fast ausschliesslich mit Krankheit und Pathogenese, da hat sie ihre Erfolge erzielt. Gesundheit und Salutogenese gehen vergessen. Man übersieht, dass Krankheiten durch die (spontane) Gesundung immer mehr oder weniger kompensiert und in Schach gehalten werden. Zudem sind pathologische Befunde häufig falsch positiv, Resultat von Zufallsfehlern und der natürlichen Variation, da es in Wirklichkeit keine feste Grenze zwischen gesund und krank gibt [2]. Auch richtig krankhafte Befunde kommen in derart vielen

\section{Médecine durable ou santé durable?}

L'Académie suisse des sciences médicales pose la question centrale de savoir s'il ne faudrait pas parler de «santé durable» plutôt que de «médecine durable». Dans les faits, la santé a toujours été l'objet de la médecine, et la médecine fondée sur les preuves l'a replacée explicitement au centre des recherches. En médecine, on pense généralement à tort que la santé n'est possible qu'en luttant contre la maladie, et qu'en soignant cette dernière, on génère automatiquement la santé.

On oublie qu'une guérison spontanée est aussi possible. La maladie se déroule dans une alternance naturelle entre pathogenèse et salutogenèse, ce qui explique qu'il n'existe en réalité aucun traitement spécifique pour des diagnostics spécifiques de maladies. Or la médecine est trop souvent dommageable lorsqu'elle ne corrige pas cette erreur. Dans la plupart des cas en effet, l'hôte décide lui-même de l'évolution de la maladie grâce à sa capacité de guérison, tandis que la conception usuelle de la médecine ignore l'hôte et pousse à lutter contre la maladie même si cela implique un surdiagnostic et un surtraitement indésirables. II est donc essentiel d'établir une théorie et une classification de l'hôte et de la guérison, pour compléter nos connaissances sur la maladie.

Fällen ohne Einschränkung der Gesundheit vor, dass Gesunde und von selber Gesundende in wachsendem Ausmass durch die Medizin krank gemacht werden - durch Überdiagnose und Übertherapie [3]. Politik und Ökonomie reagieren, indem sie die freie Ausübung solch «ungesunder» Medizin zunehmend beschränken und reglementieren wollen - meist kontraproduktiv und erfolglos, weil es das medizinische Denken ist, das korrigiert werden muss. 
Die moderne Medizin hat sich in Spezialfächer aufgeteilt, die ihre jeweilige Aufgabe darin sehen, ihre Pathologien und Krankheiten mit allen Mitteln zu bekämpfen - mit «Maximalmedizin», wie es das SAMW-Papier nennt. Zwar war die Krankheitslehre immer der alleinige Gegenstand des Medizinstudiums. Ein angstfreierer Umgang mit dem Sterben bestimmte aber früher die Praxis ebenso, und das «Patientengut» bestand zur Hauptsache aus richtig kranken Patienten mit entsprechend hohen VortestWahrscheinlichkeiten. So waren falsch positive und klinisch irrelevante Befunde noch kaum ein Problem. Dann wurden neue diagnostische Aktivitäten zunehmend auch ambulant möglich (mit entsprechend tiefen Vortest-Wahrscheinlichkeiten) und nahmen rasant zu - unter Missachtung der Regeln des Medical Decision Making [4], das die Gefahr von Überdiagnose erkennbar gemacht und böse und bedrohlich wirkende pathologische Befunde relativiert hätte. In der Euphorie über immer bessere Technologien zur Krankheitsbekämpfung wurde verkannt, dass die meisten «Erfolge» weiterhin das Ergebnis der spontanen Gesundung waren - man liess sich von der Selektion günstiger Fälle täuschen.

\section{Diskrepanz zwischen «Krankheits-Verschöne- rung» und gesundheitlichem Nutzen}

Überdies unterliegt das konventionelle medizinische Denken auch der Täuschung, die Beseitigung von Krankheiten bringe automatisch mehr Gesundheit. Mit den Glitazonen lassen sich z. B. deutlich bessere $\mathrm{HbA}_{1 \mathrm{c}}$-Werte erzielen (was sich gut verkaufen liess).
Einzelfall) liegt, wissen wir nicht. Wenn wir uns über dieses fehlende Wissen klar bleiben, können wir die ärztliche Aufmerksamkeit auf den Patienten richten und darauf, wie er die Behandlung verträgt und wie es ihm insgesamt geht. Ein «idealer» Blutdruckzielwert kommt dem medizinischen Denken entgegen, doch viele Patienten werden damit vermutlich geschädigt.

Der Surrogat-Trugschluss ist inzwischen gut dokumentiert, deshalb gelten typischerweise erst klinisch bedeutsame Krankheits-Ereignisse oder die (spezifische) Mortalität als gültige Endpunkte. Bei genauer Betrachtung sind allerdings auch sie nur ein Surrogat für einen möglichen medizinischen Erfolg. Denn die entscheidende Frage bleibt: Ist der Patient insgesamt gesünder geworden, haben sich auch Gesamtmorbidität oder Gesamtmortalität verbessert? Die Gesundheit wird noch nicht besser, wenn wir eine spezifische Todesursache erfolgreich reduzieren, der Patient aber gleich häufig stirbt - einfach an etwas anderem [8]. Eine erfolgreiche Krankheitsprävention macht nicht automatisch widerstandsfähiger und langlebiger. Dort, wo die Medizin pathologische Erscheinungen oder sogar die spezifische Mortalität reduzieren kann, wissen wir meist nicht, ob dies effektiv die Gesundheit und das Leiden insgesamt verbessert - an allen Krankheitsursachen. In dieses Bild passt, dass auch der weitverbreitete Check-up keine Senkung der Mortalität und Morbidität erzielt [9]. Er führt lediglich zu einer Zunahme von «Verlegenheits»-Hospitalisationen aufgrund von Überdiagnose.

\section{«Die entscheidende Frage bleibt: Ist der Patient insgesamt gesünder geworden?»}

Viele Diabetes-Patienten sind durch diese «Verbesserung» jedoch zu Schaden gekommen. Das zeigt sich, wenn wir statt dem Zuckerwert als Mass der Krankheit Endpunkte verwenden, die das gesundheitliche Ergehen des Patienten wiedergeben (z. B. die Mortalität) [5]. Nachdem vor gut 20 Jahren ein neues Extrasystolie-Medikament unerwartet zu einer Vervierfachung der Mortalität geführt hatte (CAST-Studie [6]), ist die grosse Diskrepanz zwischen «KrankheitsVerschönerung» und gesundheitlichem Nutzen ein Stück weit ins Bewusstsein der Medizin gelangt - als Surrogat-Trugschluss. Nur ein kleines Stück - denn auch heute noch wird z. B. propagiert, dass eine Hypertonie auf möglichst «ideale» Blutdruckwerte hinuntergedrückt werden müsse, obwohl entsprechende Studien [7] bloss zeigen, dass der gesündere Wirt noch idealer auf Blutdruckmedikamente reagiert (ein Healthy Responder Bias). Die Idee, es gäbe einen gleichen idealen Wert für alle, ist eher unbiologisch, und wo der optimale Blutdruckzielwert (im
Verbesserungen der Gesundheit können eintreten, selbst wenn Pathologien persistieren oder unbehandelt bleiben. Orthopädische Verletzungen können z. B. allein durch die natürliche Heilung wieder zu einem funktionell einwandfreien Ergebnis führen, selbst wenn grössere strukturelle Schädigungen persistieren (z.B. rupturierte Kreuzbänder [10]). Und eine Leukämie (CLL) mit den chronischen Symptomen Müdigkeit, Bauchschmerzen, Infektanfälligkeit und Schlafstörungen kann sich durch eine supportive Therapie (Kraftsuppe, Chinesische Kräutermedizin) über 3-4 Jahre klinisch markant verbessern, obwohl keine Remission der leukämischen Blutwerte stattfindet - die Leukämie bleibt, aber der Patient wird anhaltend gesund. Es gibt in der Praxis unzählige Beispiele für gesunde Verläufe bei persistierender Pathologie. Dies widerspiegelt ja nur die Natural history fast aller Krankheitsdiagnosen, die regelmässig eine breite Streuung von quasi gesunden bis zu fatalen Verläufen kennt. 
Die Tuberkulose ist vielleicht das einschlägige Beispiel, wie die Medizin gelegentlich zu einem Bewusstsein über die Rolle des Wirts gelangt ist. Man hatte erkannt, dass nicht eine variierende Virulenz oder Bösartigkeit des Krankheits-Erregers die Verschiedenartigkeit klinischer Verläufe und den drastischen Rückgang der Tuberkulose erklären, sondern Unterschiede beim Wirt, der nicht mehr Unterernährung und feuchtkalten Wohnverhältnissen ausgesetzt war. Die Medizin kam bisweilen sogar zum Schluss, dass eine Tuberkulose bei gesunden, symptomfreien Menschen am besten in Ruhe gelassen werden soll [11]. Genauso lässt die vorhandene Evidenz die Auffassung zu, dass auch bei Brustkrebs und Prostatakrebs nicht die Aggressivität und Bösartigkeit der Krebszelle den klinischen Verlauf bestimmen, sondern die Widerstandskräfte und das Gesundungsvermögen des Wirts [12]. Bösartige Pathologien bleiben in der grossen Mehrheit bis ans Lebensende unentdeckt (ausser wir sind so unklug reiche Behandlung von Krankheiten und Pathologien (allein) ein fragwürdiges Ziel darstellt.

Welche Verlaufsmöglichkeiten kennt die Natural history der vorliegenden Krankheit und wo lässt sich unser Patient einordnen? Wie gut hat er bis jetzt seine Gesundheit aufrechterhalten? Wo sind seine Stärken und wo seine Schwächen? Entspricht die Krankheitsäusserung vielleicht einem Gesundungsvorgang, der z. B. müde macht und (teil-)arbeitsunfähig (um so die Regeneration und die erneute Gesundheit zu ermöglichen)? Wie können wir eine erfolgreiche Regeneration erkennen (auch wenn diese mühsam ist und vielleicht eine ganze Weile depressiv macht)? Mit Fragen dieser Art und den entsprechenden Beobachtungen lassen sich eine Gesundungslehre und eine Klassifikation des Wirts entwickeln. Hausärzte müssen erkennen können, was im Individualfall entscheidend oder nebensächlich ist, und wann zuschauen und trösten besser ist als «medizinisch» zu intervenieren. Hausärzte müssen die

\section{«Es gibt in der Praxis unzählige Beispiele für gesunde Verläufe bei persistierender Pathologie.»}

und screenen danach). Supportive BehandlungsStrategien, die das Gesundungsvermögen und die Gesamt-Gesundheit des Patienten im Auge haben, könnten bewirken, dass ein ungünstiger Verlauf sich in einen günstigen Verlauf wendet, ohne dass die Krankheit spezifisch bekämpft wird. Es braucht mehr Forschung in diese Richtung. Eine wissenschaftliche evidenzbasierte Medizin stellt immer die Frage: Was wissen wir nicht bzw. was müssten wir eigentlich wissen? Wir müssen wissen, ob eine Massnahme die Gesundheit insgesamt stützt und mehr ist als nur Krankheitskosmetik. Nachhaltigkeit ist erst möglich, wenn wir den Gesundheits-Gewinn «messen» und im Auge behalten.

Mit der International Classification of Diseases (ICD) verwenden wir eine Pathologie-orientierte Klassifikation, bei der sich immer die Frage stellt, ob sie ein reales Patienten-Problem anzeigt oder vielmehr nur zu unnötigen oder irrelevanten Massnahmen verleitet. Alvan Feinstein hatte z. B. aufgezeigt, dass die erhaltenen Fähigkeiten des Wirts den Verlauf bei Krebskrankheiten weit besser vorhersagen können als das TNMStadium [13] - und er hat als notwendige Ergänzung eine Klassifikation des Wirts und des Krankseins (illness) gefordert [14]. Wenn wir die Fähigkeiten konsequenter zum Erfolgskriterium ärztlicher Tätigkeit machen - Alltagsfähigkeit, Arbeitsfähigkeit, Sportfähigkeit, Regenerationsfähigkeit, Konfliktfähigkeit usw. -, dann «messen» wir die Gesundheit statt die Krankheit und lernen verstehen, weshalb die erfolg-
Patienten vor zu vielen «notwendigen» Behandlungen bewahren. «Multimorbidität» ist eine Diagnose des Wirts-Zustands, nicht eine Addition spezifischer Einzelkrankheiten, die es alle vermeintlich spezifisch zu behandeln gilt. Die Abbildung 1 will schematisch zeigen, wie die Krankheit und der Wirt erst zusammen die Diagnose ausmachen.

\section{Abbildung 1}

Diagnose der Patientensituation aufgrund von Krankheit und Gesundungsvermögen.

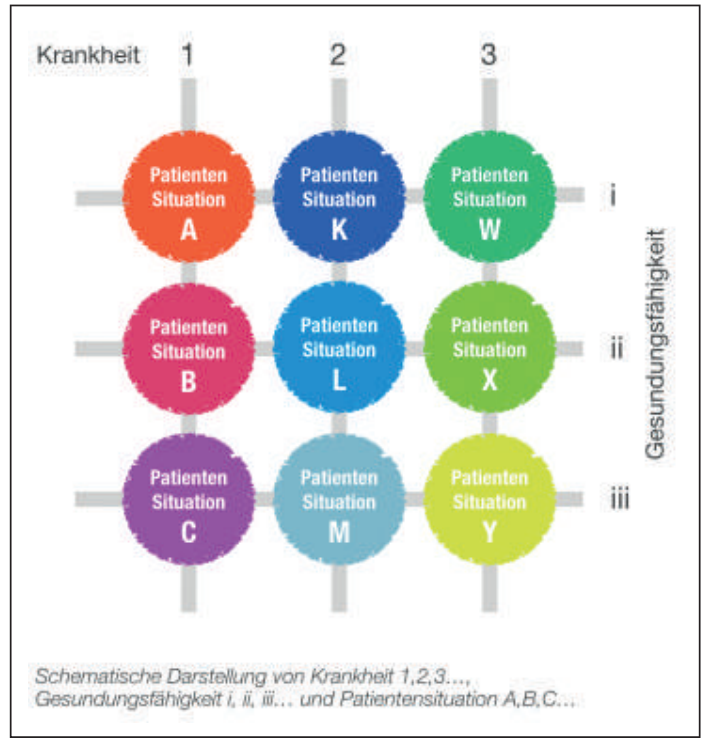


Es geht um Gesundung und ihre Klassifikation Das natürliche Wechselspiel zwischen Krankheit und Wirt, zwischen Pathogenese und Salutogenese, hat zur Folge, dass es in Wirklichkeit keine spezifischen Therapien für spezifische Krankheits-Diagnosen gibt - wenn wir einen Moment darüber nachdenken. Die Medizin schadet zu oft, wenn sie diesen Irrtum nicht korrigiert.

Seit gut 30 Jahren existiert schon eine International Classification of Impairments, Disabilities and Handicaps (ICIDH), die vor zehn Jahren zur International Classification of Functioning, Disability and Health (ICF) weiterentwickelt worden ist. Gute Anhaltspunkte für eine Klinik und Klassifikation der Gesundung kann mitunter auch die Komplementärmedizin bieten. In der Altchinesischen Medizin etwa besteht die Diagnose darin, die Funktionsfähigkeit der einzelnen «Organe» und deren Zusammenwirken zu bestimmen, damit der Ursprung der mannigfachen Symptome und Beschwerden erkennbar wird. Die Frage ist immer: Wo in der Organ-Abfolge ist die Gesundungskraft erschöpft oder blockiert, wo hat es angefangen? Zeigt sich im jeweiligen pathologischen Vorgang ein gesunder Aufräumprozess oder eine krankhafte Dekompensation? Auf diese Weise wird z.B. erkennbar, dass Schübe bei MS-Patienten primär einen Gesundungsvorgang darstellen [15], was gut erklärt, weshalb Immunmodulatoren keinen wahren Nutzen zeigen [16]. Auch die von Feinstein entwickelte Klinimetrie, welche die Frage nach dem vom Patienten erlebten Nutzen und dessen Bemessung stellt [17], bietet eine gute methodische Grundlage für die anstehende Aufgabe: Die Beschreibung des Wirts und der Gesundung und deren Klassifikation.

Viele systematische Analysen des gesundheitlichen Nutzens medizinischer Massnahmen sind in den letzten 20 Jahren geleistet worden - etwa durch die Cochrane Collaboration. Die «Stiftung Paracelsus heute» will darüber hinaus eine klinische Lehre der Gesundung voranbringen - als Ergänzung zur Krankheitslehre. Sie will eine zeitgemässe Korrektur des medizinischen Denkens fördern und dem eigenen Erkennen und Verstehen Raum ermöglichen und so Mut und Eigenverantwortung von Arzt und Patient unterstützen. Eine geeignete Plattform soll die Verschiedenartigkeit ärztlicher Vorgehensweisen sichtbar machen - und so Ängste abbauen, wenn es gilt, unkonventionell und innovativ zu handeln und auf nicht nachhaltige und unnütze Medizin zu verzichten.

\section{Literatur}

1 Nachhaltige Medizin - Positionspier der Schweizerischen Akademie der Medizinischen Wissenschaften. Schweiz Ärztezeitung 2012; 93(45):1645-53. www.saez.ch/docs/saez/2012/45/de/SAEZ-01056.pdf

2 Schmidt J. Das Praxislabor: Die scholastischen Täuschungen der «Qualitätskontrolle».
Schweiz Ärztezeitung. 2012; 9348):1796-9. www.saez.ch/docs/saez/2012/48/fr/BMS-00954.pdf

3 Welch HG, Schwartz L, Woloshin Steve. Overdiagnosed: Making People Sick in the Pursuit of Health. Beacon Press; 2012.

4 Sox HC, Higgins MC, Blatt MA, Marton KI. Medical Decision Making. Butterworth-Heinemann; 1988.

5 Gysling E. Diabetes zum Beispiel (Editorial). pharmakritik 2010; 32: Nr. 3.

6 Cardiac Arrhythmia Suppression Trial (CAST) Investigators. Preliminary report: effect of encainide and flecainide on mortality in a randomized trial of arrhythmia suppression after myocardial infarction. N Engl J Med. 1989;321:406-12.

7 Weber MA, Bakris GL, Hester A et al. Systolic blood pressure and cardiovascular outcomes during treatment of hypertension. Am J Med. 2013; 26:501-8.

8 Schmidt JG. Evidenz-basierte Medizin - Modebegriff oder zukunftsweisend? Vortrag am RVK-Forum 2013 Luzern. www.rvkforum.ch/sites/default/files/ referenten-praesentationen/praesentation-schmidt.pdf

9 Krogsbøll LT, Jørgensen KJ, Larsen CG, Gøtzsche PC. General health checks in adults for reducing morbidity and mortality from disease. The Cochrane Library. October 2012

10 Medical Board. Ruptur des vorderen Kreuzbandes: operative oder konservative Behandlung? Bericht vom 30. Juni 2009 (Gesundheitsdirektion des Kantons Zürich). www.medical-board.ch/fileadmin/docs/ public/mb/Fachberichte/2009_06_30_Ruptur_Kreuzband_Bericht.pdf

11 Smith BM, Schwartzman K, Bartlett G, et al. Adverse events associated with treatment of latent tuberculosis in the general population. CMAJ. 2011;183:E173-9.

12 Schmidt JG. Ist Verzicht immer Nihilismus? Über das Handeln aufgrund rationaler Erkenntnisse. Vortrag am Deutschen Internistenkongress. In: Köbberling J (Hrsg.). Zeitfragen der Medizin. Berlin Heidelberg: Springer-Verlag; 1997. S.115-25. www.paracelsus-heute. $\mathrm{ch} / \mathrm{cms} /$ literatur/PDF/Springer1997.pdf

13 Feinstein AR. The hard data creed in current clinical practice: Its spurious validity and the challenge to define meaningful clinical variables. In: Schmidt JG, Steele RE, eds. Kritik der medizinischen Vernunft: Schritte zu einer zeitgemässen Medizin - Ein Lesebuch. Mainz: Kirchheim-Verlag; 1994; S. 210-8. www. paracelsus-heute.ch/cms/literatur/011_wiss_einsiedler_ symposien/PDF_1_symposium/81Feinstein.pdf

14 Feinstein AR. Why do we need clinical epidemiology? A practice-oriented clinical science. In: Schmidt JG, Steele RE (Hrsg.). Kritik der medizinischen Vernunft: Schritte zu einer zeitgemässen Medizin - Ein Lesebuch. Mainz: Kirchheim-Verlag, 1994; 233-43. www. paracelsus-heute.ch/cms/literatur/011_wiss_einsiedler symposien/PDF_1_symposium/84Feinstein.pdf

15 Schmidt JG. Chronische Krankheiten und der Wirt Gesundung am Beispiel von Patienten mit Multipler Sklerose. Schweiz. Z. Ganzheitsmed. 2013;25:296-303. www.paracelsus-heute.ch/cms/literatur/PDF/SZG_ MS.pdf

16 Rice GPA, Incorvaia B, Munari LM, et al. Interferon in relapsing-remitting multiple sclerosis (Review). The Cochrane Library. 2009; Issue 1.

17 Feinstein AR. Clinimetrics. Yale University Press; 1987. 